\title{
Microneurosurgical removal of thalamic lesions: surgical results and considerations from a large, single-surgeon consecutive series
}

\author{
Carlo Serra, MD, ${ }^{1,3}$ Hatice Türe, MD, ${ }^{2}$ Cumhur Kaan Yaltırık, MD, ${ }^{1}$ Mehmet Volkan Harput, MD, ${ }^{1}$ and \\ Uğur Türe, MD1
}

Departments of ${ }^{1}$ Neurosurgery and ${ }^{2}$ Anesthesiology, Yeditepe University School of Medicine, Istanbul, Turkey; and ${ }^{3}$ Department of Neurosurgery, Clinical Neuroscience Centre, University Hospital Zürich, University of Zürich, Switzerland

\begin{abstract}
OBJECTIVE The object of this study was to present the surgical results of a large, single-surgeon consecutive series of patients who had undergone transcisternal (TCi) or transcallosal-transventricular (TCTV) endoscope-assisted microsurgery for thalamic lesions.
\end{abstract}

METHODS This is a retrospective study of a consecutive series of patients harboring thalamic lesions and undergoing surgery at one institution between February 2007 and August 2019. All surgical and patient-related data were prospectively collected. Depending on the relationship between the lesion and the surgically accessible thalamic surfaces (lateral ventricle, velar, cisternal, and third ventricle), one of the following surgical TCi or TCTV approaches was chosen: anterior interhemispheric transcallosal (AIT), posterior interhemispheric transtentorial subsplenial (PITS), perimedian supracerebellar transtentorial (PeST), or perimedian contralateral supracerebellar suprapineal (PeCSS). Since January 2018, intraoperative MRI has also been part of the protocol. The main study outcome was extent of resection. Complete neurological examination took place preoperatively, at discharge, and 3 months postoperatively. Descriptive statistics were calculated for the whole cohort.

RESULTS In the study period, 92 patients underwent surgery for a thalamic lesion: 81 gliomas, 6 cavernous malformations, 2 germinomas, 1 metastasis, 1 arteriovenous malformation, and 1 ependymal cyst. In none of the cases was a transcortical approach adopted. Thirty-five patients underwent an AIT approach, 35 a PITS, 19 a PeST, and 3 a PeCSS. The mean follow-up was 38 months (median 20 months, range 1-137 months). No patient was lost to follow-up. The mean extent of resection was $95 \%$ (median 100\%, range $21 \%-100 \%$ ), and there was no surgical mortality. Most patients $(59.8 \%)$ experienced improvement in their Karnofsky Performance Status. New permanent neurological deficits occurred in 8 patients $(8.7 \%)$. Early postoperative ( $<3$ months after surgery) problems in CSF circulation requiring diversion occurred in 7 patients (7.6\%; $6 / 7$ cases in patients with high-grade glioma).

CONCLUSIONS Endoscope-assisted microsurgery allows for the removal of thalamic lesions with acceptable morbidity. Surgeons must strive to access any given thalamic lesion through one of the four accessible thalamic surfaces, as they can be reached through either a TCTV or TCi approach with no or minimal damage to normal brain parenchyma. Patients harboring a high-grade glioma are likely to develop a postoperative disturbance of CSF circulation. For this reason, the AIT approach should be favored, as it facilitates a microsurgical third ventriculocisternostomy and allows intraoperative MRI to be done.

https://thejns.org/doi/abs/10.3171/2020.6.JNS20524

KEYWORDS microneurosurgery; thalamic cavernous malformation; thalamic glioma; thalamic lesions; thalamus; oncology

ABBREVIATIONS AIT = anterior interhemispheric transcallosal; CC = corpus callosum; EOR = extent of resection; GTR = gross-total resection; IC = internal capsule; ioUS = intraoperative ultrasonography; KPS = Karnofsky Performance Status; m3VC = microsurgical 3VC; NTR = near-total resection; PeCSS = perimedian contralateral supracerebellar suprapineal; PeST = perimedian supracerebellar transtentorial; PITS = posterior interhemispheric transtentorial subsplenial; STR = subtotal resection; TCi = transcisternal; TCTV = transcallosal-transventricular; VPS = ventriculoperitoneal shunt; 3T-ioMR = intraoperative 3-T MRI; 3VC = third ventriculocisternostomy.

SUBMITTED February 19, 2020. ACCEPTED June 1, 2020.

INCLUDE WHEN CITING Published online October 2, 2020; DOI: 10.3171/2020.6.JNS20524. 
$\mathrm{T}$ HE deep location of the thalamus and its proximity to vital neural and vascular structures pose a formidable challenge to surgeons willing to pursue thalamic surgery, ${ }^{1-4}$ and a conservative attitude toward such lesions dominates the literature. A biopsy followed by adjuvant therapy is the favored approach for thalamic tumors, ${ }^{5}$ whereas thalamic cavernous malformations are removed at only highly selected centers. ${ }^{6-9}$

Reaching the thalamus is a demanding task, as almost $50 \%$ of the thalamic surface is covered by the internal capsule (IC) laterally and by the hypothalamus and the mesencephalon anteroinferiorly. Consequently, surgical approaches through a lateral transcortical pathway damage eloquent normal brain parenchyma. Notwithstanding these data, the literature indicates that the transcortical approaches are widely adopted for these lesions..$^{6-8,10-33}$ However, a considerable portion of the thalamus surface abuts cisternal or ventricular spaces and thus can be reached through either a transcisternal (TCi) or transcallosaltransventricular (TCTV) approach with no or minimal damage to normal brain parenchyma. Clearly identifiable anatomical landmarks allow segmentation of this surface into four surgically accessible thalamic surfaces: lateral ventricle, velar, cisternal, and third ventricle (Fig. 1). ${ }^{2}$ Most lesions within the thalamus can be safely reached through one of these surfaces. And each of these surfaces can in turn be reached by one or more of the following TCTV or TCi approaches (Fig. 2): anterior interhemispheric transcallosal (AIT), posterior interhemispheric transtentorial subsplenial (PITS), perimedian supracerebellar transtentorial (PeST), and perimedian contralateral supracerebellar suprapineal (PeCSS). The lateral ventricular and velar surfaces are best reached through the AIT approach, the cisternal surface through the PeST or PITS approach, and the third ventricle surface through the PeCSS approach. ${ }^{1,34}$

The aim of this study is to present the surgical results of a large, single-surgeon, consecutive series of patients who underwent endoscope-assisted microsurgery for a thalamic lesion according to the abovementioned principles. Exemplary cases are illustrated together with considerations of the merits and pitfalls of the different approaches.

\section{Methods}

\section{Patient Data and Outcome Assessment}

Prospectively collected data on patients undergoing surgery at our institution for a thalamic lesion between February 2007 and August 2019 were retrospectively reviewed. Preoperative and immediate postoperative $(<24$ hours) 3-T brain MRI scans were done according to a standard protocol. Preoperative and 3-month postoperative tractography was also part of the protocol. Intraoperative 3-T MRI (3T-ioMR) was introduced into our clinical routine in January 2018. Intraoperative neuromonitoring was routinely performed (evoked motor and somatosensory potentials as well as subcortical stimulation).

The extent of resection (EOR) was assessed volumetrically with OsiriX software. Manual segmentation of a lesion's volume occurred by comparing pre- and postoperative imaging depending on the histopathology of the lesion. Gross-total resection (GTR) was defined as an EOR
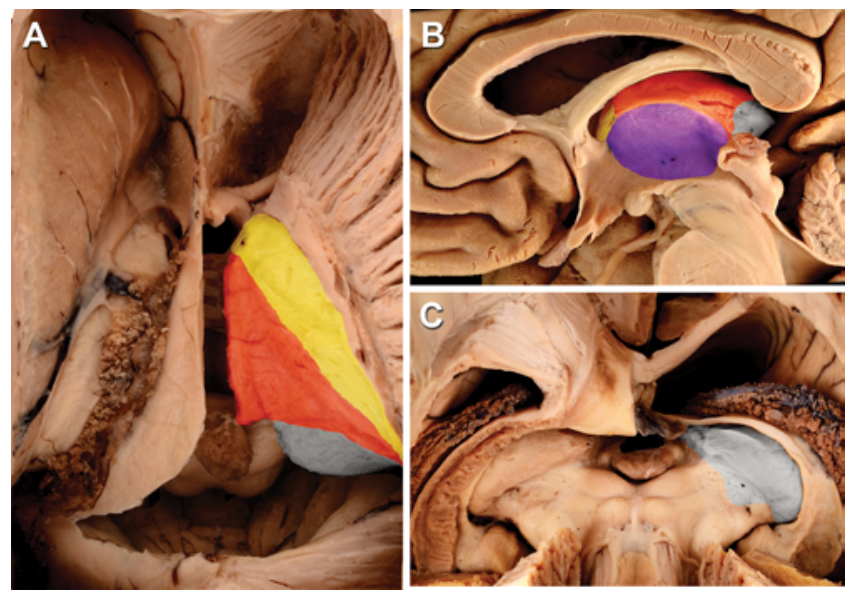

FIG. 1. Outline of the free thalamic surfaces. Red indicates the velar surface; purple, the third ventricle surface; gray, the cisternal surface; and yellow, the lateral ventricle surface. Reprinted from World Neurosurgery, 97, Serra C, Türe U, Krayenbühl N, Şengül G, Yaşargil DCH, Yaşargil $M G$, Topographic classification of the thalamus surfaces related to microneurosurgery: a white matter fiber microdissection study, 438-452, Copyright 2017, with permission from Elsevier.

$>99 \%$, near-total resection (NTR) as an EOR between 95\% and 99\%, and subtotal resection (STR) as an EOR $<95 \%$.

All patients underwent a neurological examination at admission, immediately after surgery, at discharge, and at 3 months postoperatively. Follow-ups were repeated thereafter depending on the histopathology of the lesion. Preoperatively cooperative patients harboring a lesion in the pulvinar underwent an ophthalmological examination including visual acuity and visual field assessment. We defined permanent neurological deficits as new surgeryrelated neurological deficits present at the 3-month postoperative follow-up.

Patient data are reported according to common descriptive statistics. Written informed consent was obtained for all patients. The study was performed according to the ethical standards of the Declaration of Helsinki and approved by our institutional review committee.

\section{Surgical Techniques}

All patients were operated on through either a TCTV or a TCi microsurgical approach performed under the operating microscope with endoscopic assistance. Several variables determined the choice of surgical approach: suspected histopathology; location, size, and extension of the lesion; relationship with the four surgically accessible thalamic surfaces; relationship of the lesion to the corpus callosum (CC); lesion relationship with the deep venous system and the tentorial angle; patient age; presence of disturbances in the circulation of CSF; need for 3T-ioMR; and presence of a patent foramen ovale with a spontaneous shunt.

Preoperative MRI scans were loaded into the OsiriX software $^{35}$ to obtain a 3D reconstruction of the cerebral surface, vessels, and targeted lesion. Placement of the craniotomy was decided based on the superficial venous 


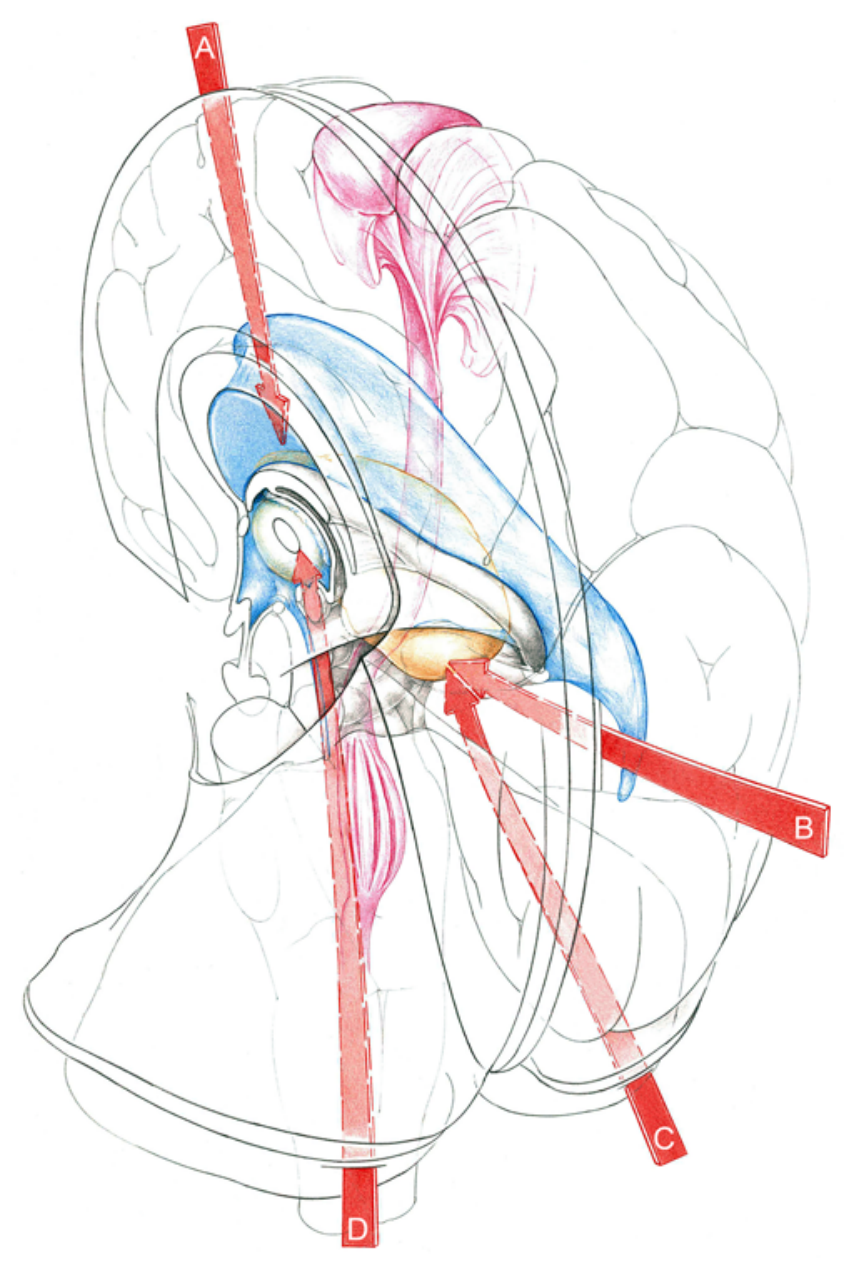

FIG. 2. Illustration of the available TCi and TCTV approaches. The thalamus of the right hemisphere is depicted in orange from a superoposteromedial oblique view. The left hemisphere has been removed. The lateral and third ventricles are visible in blue. The pyramidal tract appears in pink as it runs from the precentral gyrus through the IC (thus lateral to the thalamus) and the crus cerebri toward the periphery. As detailed in the text, the thalamus can be approached from anterior to posterior via the following approaches: $A$, anterior interhemispheric transcallosal (AIT); B, posterior interhemispheric transtentorial subsplenial (PITS); C, perimedian supracerebellar transtentorial (PeST); and D, perimedian contralateral supracerebellar suprapineal (PeCSS). These approaches run along either the interhemispheric or transverse fissure; that is, the falx or the tentorium, respectively, which are also shown in the illustration. Copyright Uğur Türe. Published with permission.

anatomy of the patient, crucial for both interhemispheric approaches (AIT and PITS).

The AIT approach was performed as previously described, ${ }^{6,36}$ on the right side given the surgeon's (U.T.) dominant hand. The craniotomy extends contralaterally so that the surgeon can switch to a left-sided approach if the venous anatomy requires it. A callosotomy is done under intraoperative ultrasonography (ioUS) guidance. Once the CC is reached, a cottonoid is placed over the tentative callosotomy point, and its position relative to the $\mathrm{CC}$ and the lesion is studied under ioUS. ${ }^{10}$ Callosal fibers are separated by a few millimeters between the two pericallosal arteries, and the targeted lateral ventricle is entered.
The endoscope is inserted to explore the intraventricular anatomy and appropriately enlarge the callosotomy (maximum $8-10 \mathrm{~mm}$ ). The foramen of Monro, choroid plexus, tenia choroidea, stria terminalis, and thalamostriate vein should be promptly identified. These landmarks help in defining the borders of the lateral ventricular surface of the thalamus and planning access to the velar surface or to the third ventricle's surface if needed. If the approach angle allows it, a transforaminal microsurgical third ventriculocisternostomy (m3VC) is performed within the same surgery. For an illustrative case, see Fig. 3 and Video 1.

VIDEO 1. Clip demonstrating the radical resection of a large tumor originating from the left thalamus and dislocating the surrounding structures, causing hydrocephalus. The tumor was removed via an endoscope-assisted AIT approach combined with m3VC. The surgery was uneventful, and the patient experienced no postoperative deficits. This is the same case featured in Fig. 3. Copyright Uğur

Türe. Published with permission. Click here to view.

The PITS approach is directed to the cisternal surface, as previously described by our group..$^{38}$ The approach can be performed with the patient in the semisitting, lateral, or prone oblique position. The latter has become standard in our practice, as it allows 3T-ioMR to be done. The surgical corridor is directed through the interhemispheric fissure along the medial surface of the cuneus, posterior to the parietooccipital ascending vein, aiming at the splenium. Once reached, the arachnoid of the callosal and subsplenial cisterns, as well as that around the deep venous system, is opened to release CSF. Usually this maneuver allows considerable gravity relaxation of the ipsilateral hemisphere within a few minutes. At this point, the tentorium should be cut to achieve the required surgical exposure. A 2 -cm-long incision perpendicular to the tentorial hiatus is usually sufficient.

The edges of the divided tentorium are retracted and fixed at the falx with absorbable sutures. The tentorial cut should be done under Doppler guidance, as the transition between the falx and tentorium is not sharply defined and the sinus rectus cannot be safely localized, so the surgeon can rely on anatomical data only. The major venous lacunae of the tentorium should also be spared. The third ventricle can also be reached through this approach, and an $\mathrm{m} 3 \mathrm{VC}$ through the lamina terminalis is also possible. For an illustrative case, see Fig. 4.

The PeST approach is a variation of the superior median supracerebellar approach. ${ }^{6}$ The patient is placed in a semisitting position at $25^{\circ}-30^{\circ}$ unless a spontaneous rightleft shunt is demonstrated by echocardiography. A linear median occipital incision is made, and an asymmetrical perimedian craniotomy is performed via three burr holes (two on the transverse sinus, one about $2 \mathrm{~cm}$ above it). The craniotomy is relatively large and encompasses both sides (hence the name "perimedian"), the transverse sinus, and the torcular herophili, but it extends asymmetrically more on the side of the pathology. In this way, virtually any tentorial vein impeding the approach toward the thalamus can be circumvented and spared. Under the operating microscope, the dura mater is incised at the caudal end of the exposure to release CSF from the cisterna magna. A second curvilinear incision is then made about $15 \mathrm{~mm}$ under the transverse sinus. In this way, the cerebellar pa- 

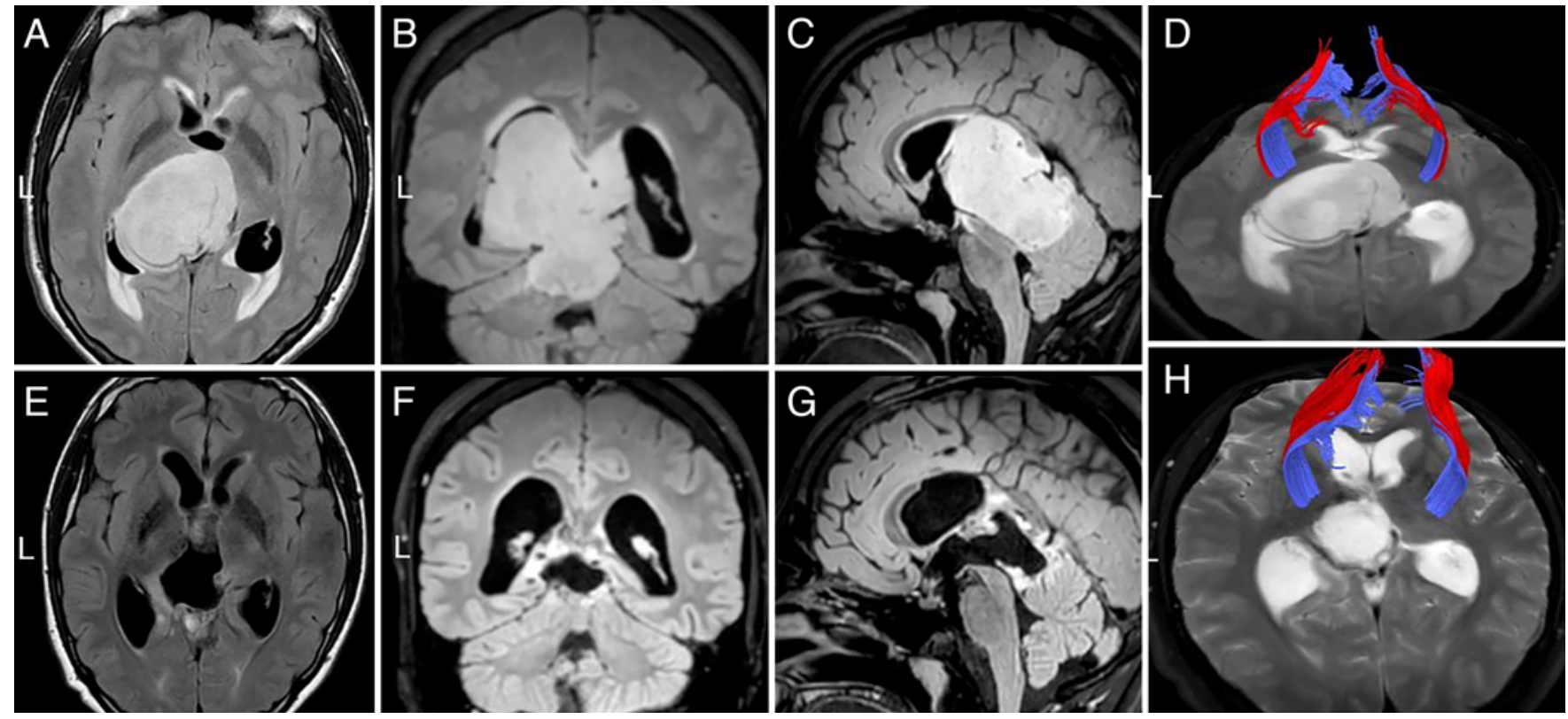

FIG. 3. Images obtained in a 14-year-old boy with complaints of headache, recurrent vomiting, and diplopia in the previous week. At referral to our institution, he presented with mild cognitive deficits (Glasgow Coma Scale score 14) and diplopia in all gaze directions. Neuroimaging showed a large lesion originating from the left thalamus and dislocating the surrounding structures, causing hydrocephalus. The tumor was removed via an endoscope-assisted AIT approach combined with m3VC. Surgery was uneventful, and the patient experienced no postoperative deficits. Postoperative MRI confirmed radical resection. Histopathological examination revealed an anaplastic astrocytoma (WHO grade III), and the patient underwent adjuvant therapy. Preoperative axial (A), coronal (B), and sagittal (C) FLAIR MRI as well as a 3D reconstruction image (D) of the sensory (red) and motor (blue) fiber components of the IC. Postoperative axial $(\mathbf{E})$, coronal $(\mathbf{F})$, and sagittal $(\mathbf{G})$ FLAIR MRI and 3D reconstruction $(\mathbf{H})$ showing results. $L=$ the patient's left.

renchyma relaxes, and the virtual space between the tentorium and cerebellar surface opens, permitting atraumatic access to the cisternal surface of the thalamus through the transverse fissure. The tentorium is then cut longitudinally along the direction of the approach. After incision of the tentorium, the endoscope is inserted up to the tentorial incisura just before attacking the lesion in order to visualize all relevant anatomical relationships. If needed, the ipsilateral mediobasal temporal region can also be reached within the same procedure. For an illustrative case, see Fig. 5 and Video 2.

VIDEO 2. Clip demonstrating the radical removal of a left-sided thalamic tumor located at the pulvinar. A PeST approach is used to reach the tumor. The operation was uneventful and GTR could be achieved. This is the same case featured in Fig. 5. Copyright Uğur Türe. Published with permission. Click here to view.

The PeCSS approach is used for lesions on the third ventricle surface of the thalamus..$^{39}$ The skin incision and the craniotomy are done as in the PeST approach, with the only difference being that the asymmetrical craniotomy extends more toward the side contralateral to the lesion. The supracerebellar route is then exploited to reach and open the quadrigeminal cistern and expose the trochlear nerve, tectum, and pineal body. The arachnoidal dissection is then extended superoanteriorly into the third ventricle via a suprapineal pathway. No tentorial incision is needed. The endoscope is inserted to explore the anatomy of the third ventricle, particularly its inferior portion, which is the most difficult point to be visualized and reached under the microscope. For this purpose, the use of curved suction tools under endoscopic guidance (angled optic) is of great help for removing lesions in this region. For an illustrative case, see Fig. 6.

\section{Results}

In the study period, 96 patients were referred to our institution for surgery for a thalamic lesion. Four patients underwent stereotactic biopsy (3 lymphoma patients and 1 glioblastoma patient with medical contraindications for open surgery), and 92 underwent open surgery aimed at the maximum possible resection. Fifty-two patients had a high-grade lesion and 40 had a low-grade lesion. The tumor was unilateral unifocal in 87 patients and multifocal in the remainder ( 2 cases bilateral, 2 cases thalamic and temporal mediobasal, 1 case thalamic and in the fourth ventricle). In none of the cases was a transcortical approach adopted. Thirty-five patients underwent an AIT approach, 35 a PITS, 19 a PeST, and 3 a PeCSS. The mean follow-up was 38 months (median 20 months, range 1-137 months). Detailed pre- and postoperative clinical data are reported in Tables 1 and 2.

\section{Extent of Resection and Clinical Outcome}

GTR was achieved in 61/92 patients (66\%), NTR in 24/92 (26\%), and STR in 7/92 (8\%). 3T-ioMR was performed in 9/16 cases after its introduction at our institution. In $3 / 9$ cases its use led to further resection, and in $1 / 3$ 

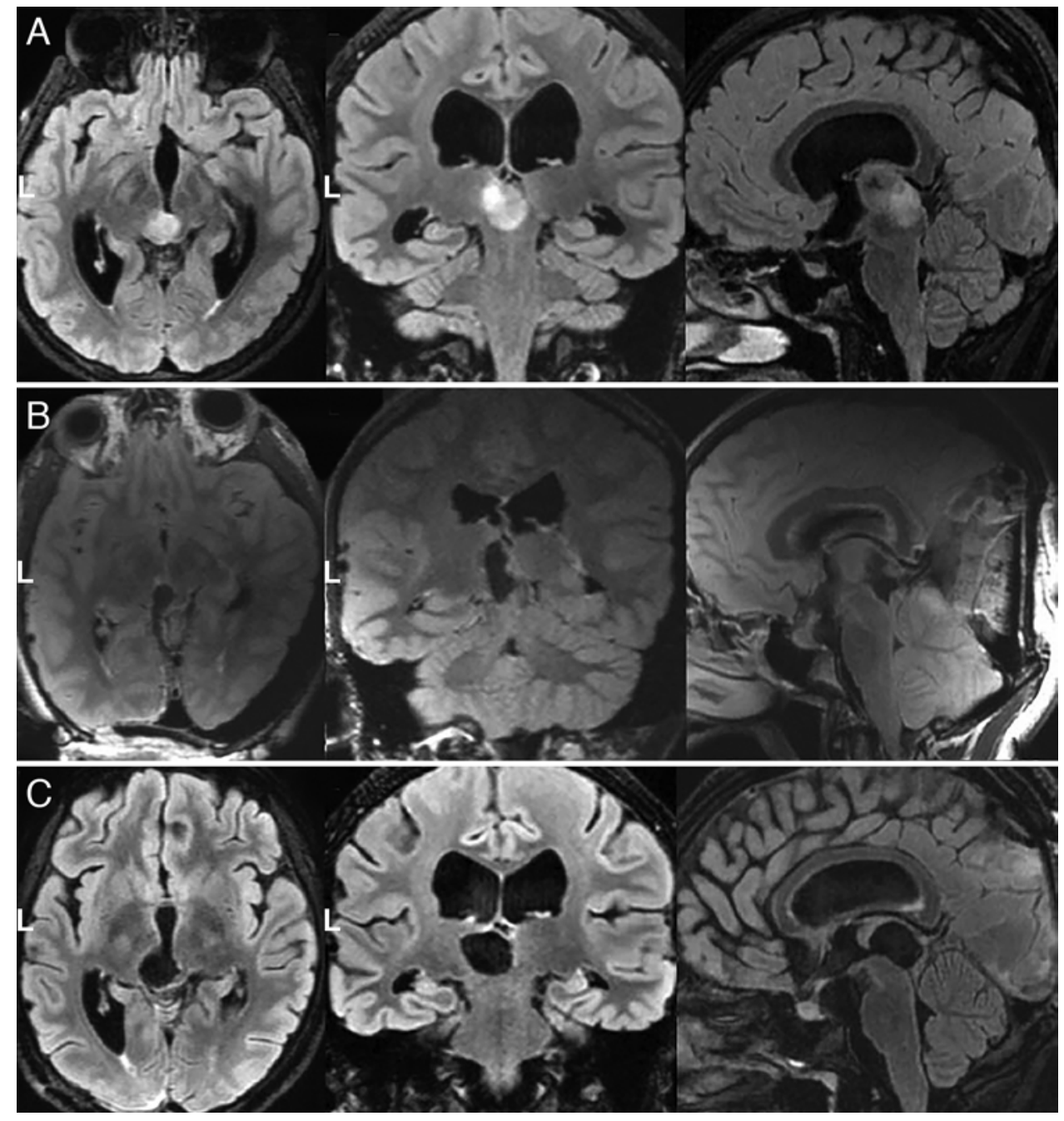

FIG. 4. Images obtained in a 13-year-old girl who had presented with severe headache at another institution. CT performed there showed triventricular hydrocephalus, which prompted an emergency endoscopic 3VC. The patient was then referred to our institution where MRI showed a lesion in the left pulvinar responsible for the subocclusive hydrocephalus. The radiological appearance of the lesion indicated a posterior approach. Given the relation between the splenium and tumor, either a PeST or PITS approach would have been possible. However, a PITS approach would allow a superior view into the aqueduct. The patient was placed in the prone oblique position to allow 3T-ioMR. Complete resection was achieved at the cost of a transient upward gaze palsy only, which resolved 2 months after surgery. Histopathological analysis revealed a WHO grade II IDH-wildtype glioma, and the patient underwent adjuvant therapy. A: Preoperative axial, coronal, and sagittal FLAIR MRI. B: Intraoperative FLAIR MRI. C: Postoperative MRI.

cases it led to GTR. In 6/9 cases no further resection was pursued, with the prefixed target having been achieved. The average EOR was 95\% (median 100\%, range 21\%$100 \%)$. The mean preoperative lesion volume was 24.8 $\mathrm{cm}^{3}$ (median $15.3 \mathrm{~cm}^{3}$, range $0.42-129.2 \mathrm{~cm}^{3}$ ), and the mean postoperative volume was $2.2 \mathrm{~cm}^{3}$ (median $0 \mathrm{~cm}^{3}$, range $0.0-71.89 \mathrm{~cm}^{3}$ ). Detailed resection results are summarized in Table 2.

Most patients (59.8\%) experienced clinical improvement after surgery as measured by the Karnofsky Performance Status (KPS; Table 1). There was no surgical mortality, and only one major surgical morbidity in the series. One patient developed a severe CSF disturbance postoperatively, which led to 5 subsequent operations attempting to restore his CSF circulation. Consequently, the patient could not start the required adjuvant therapy and died 3 months after surgery. Six patients $(6.5 \%)$ had a permanent deterioration in their KPS, whereas 8 patients $(8.7 \%)$ had new permanent hemiparesis. Most complications occurred in patients harboring a high-grade lesion (4/6 with KPS deterioration and 6/8 with new permanent hemiparesis). Medical complications occurred in 14 patients (15.2\%; 6 pneumonia, 2 deep venous thrombosis, 6 metabolic), although none of the complications resulted in permanent morbidity. Intraoperative diabetes insipidus (remittent after desmopressin) developed in 15 patients. Complications requiring surgery (other than CSF disturbances) occurred in 2 patients (1 wound revision, 1 epidural hematoma).

Complete pre- and postoperative visual field examination data were available in 22 patients. Thirteen (59.1\%) 

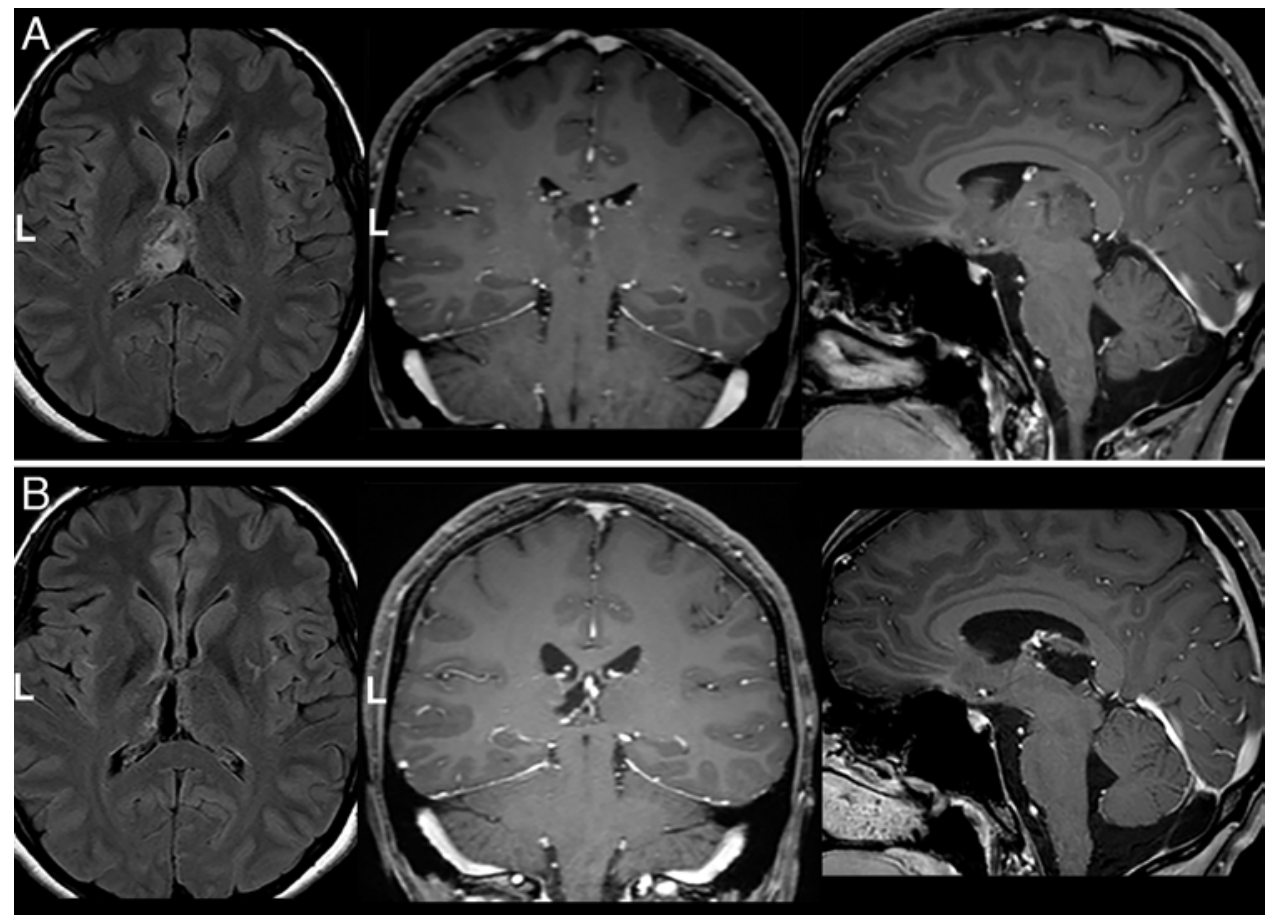

FIG. 5. Imaging obtained in a 20 -year-old female who had been experiencing headache over the previous 2 months. She eventually underwent MRI, which showed a lesion in the left pulvinar. The anatomical relationship between the lesion and the splenium prompted a PeST approach with the patient in a semisitting position. The operation was uneventful, and GTR could be achieved (as shown on postoperative MRI). At histological examination, the lesion proved to be an oligoastrocytoma (WHO grade II not otherwise specified). Thus, no adjuvant therapy was given. A: Preoperative axial FLAIR and coronal and sagittal gadolinium-enhanced T1-weighted MRI. B: Postoperative imaging showing the result.
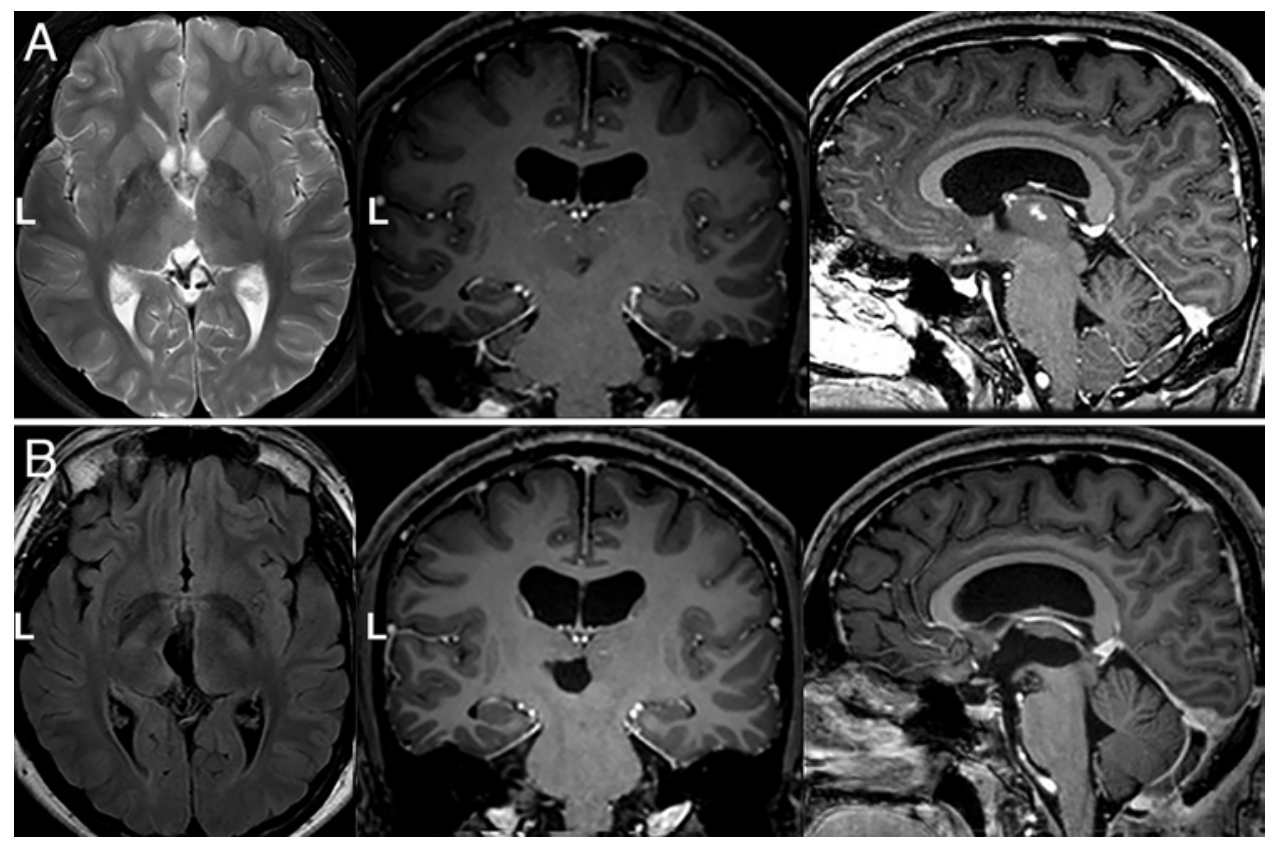

FIG. 6. Images obtained in a 16-year-old male who had been experiencing headache for 4 years. His MRI showed a lesion originating from the third ventricle surface of the thalamus. He had no deficit on neurological examination and a KPS of 100 . He underwent an endoscopic 3VC with biopsy at another institution and was then referred to us for further treatment. Appropriate neuroradiological imaging showed evidence of healthy thalamic tissue interposed between the lesion and the lateral ventricle as well as the cisternal surfaces, thus prompting the choice of a PeCSS approach with the patient in a semisitting position. Postoperative MRI confirmed GTR. Postoperatively, the patient had a transient upward gaze palsy, which resolved in 2 months. Histopathological examination revealed a diffuse astrocytoma of WHO grade II. No adjuvant therapy was given. A: Preoperative axial, coronal, and sagittal MRI. B: Postoperative imaging. 
TABLE 1. Summary of relevant clinical variables for 92 patients with thalamic lesions

\begin{tabular}{|c|c|}
\hline Variable & Value \\
\hline \multicolumn{2}{|l|}{ Sex, no. $(\%)$} \\
\hline M & $57(62.0)$ \\
\hline $\mathrm{F}$ & $35(38.0)$ \\
\hline Mean age in yrs (median, range) & $33.4(30,5-71)$ \\
\hline \multicolumn{2}{|l|}{ Age group, no. (\%), LGG, HGG } \\
\hline Pediatric: age $<18$ yrs & $22(23.9), 15,7$ \\
\hline Adults: age $\geq 18$ yrs & 70 (76.1), 25, 45 \\
\hline \multicolumn{2}{|l|}{ Side of surgery, no. (\%) } \\
\hline Rt & $50(54.3)$ \\
\hline Lt & $42(45.7)$ \\
\hline \multicolumn{2}{|c|}{ No. of operations/patient for oncological purposes } \\
\hline 1 & 78 \\
\hline 2 & 10 \\
\hline 3 & 3 \\
\hline 4 & 1 \\
\hline \multicolumn{2}{|l|}{ Clinical outcome } \\
\hline \multicolumn{2}{|l|}{ Median KPS (range) } \\
\hline At admission & $80(30-90)$ \\
\hline At discharge & $70(30-100)$ \\
\hline At 3-mo FU & $90(50-100)$ \\
\hline \multicolumn{2}{|l|}{ KPS deterioration, no. (\%) } \\
\hline At discharge & $29(31.5)$ \\
\hline At 3-mo FU & $6(6.5)$ \\
\hline \multicolumn{2}{|l|}{ KPS improvement, no. (\%) } \\
\hline At discharge & $19(20.7)$ \\
\hline At 3-mo FU & $55(59.8)$ \\
\hline Medical complication, no. (\%) & $14(15.2)$ \\
\hline \multicolumn{2}{|l|}{ Neurological outcome } \\
\hline \multicolumn{2}{|l|}{ New hemiparesis, no. (\%) } \\
\hline At discharge & $20(21.7)$ \\
\hline At 3-mo FU & $8(8.7)$ \\
\hline \multicolumn{2}{|c|}{ Improvement of hemiparesis, no./total no. (\%) } \\
\hline At discharge & $4 / 29(13.8)$ \\
\hline At 3-mo FU & $18 / 29(62.1)$ \\
\hline Hemiballismus (transient), no. (\%) & $1(1.1)$ \\
\hline Upward gaze palsy, no. (\%) & $3(3.3)$ \\
\hline Mean ICU LOS, days (median, range) & $2.7(1,1-117)$ \\
\hline
\end{tabular}

experienced postoperative visual field improvement, 4 (18.2\%) had no significant change, and 5 (22.7\%) had deterioration. Four of these latter 5 patients had undergone a PITS approach.

\section{CSF Disturbances}

Seventeen patients $(18.5 \%)$ presented preoperatively with clinically relevant hydrocephalus and had a form of preoperative CSF drainage. Five (5.4\%) had external ventricular drainage ( $2 / 5$ drains placed at another institution),
TABLE 2. Resection results and clinical outcome according to histopathology and surgical approach

\begin{tabular}{|c|c|c|c|c|}
\hline \multirow[b]{2}{*}{ Histopathology } & \multicolumn{4}{|c|}{ Approach } \\
\hline & AIT & PITS & PeST & PeCSS \\
\hline \multicolumn{5}{|l|}{ Glioma WHO grade I $(n=13)^{*}$} \\
\hline No. of patients & 6 & 4 & 1 & 2 \\
\hline EOR & $92 \%$ & $100 \%$ & $100 \%$ & $100 \%$ \\
\hline Mean preop lesion vol $\left(\mathrm{cm}^{3}\right)$ & 19.3 & 3.3 & 11.2 & 2.7 \\
\hline Mean residual lesion vol $\left(\mathrm{cm}^{3}\right)$ & 1.4 & 0 & 0 & 0 \\
\hline No. of patients w/ ND at 3-mo FU & 0 & 0 & 0 & 0 \\
\hline $\begin{array}{l}\text { No. of patients w/ KPS deteriora- } \\
\text { tion at } 3-\mathrm{mo} \text { FU }\end{array}$ & 0 & 0 & 0 & 0 \\
\hline \multicolumn{5}{|l|}{ Glioma WHO grade II $(n=17) \dagger$} \\
\hline No. of patients & 3 & 6 & 7 & 1 \\
\hline EOR & $70 \%$ & $76 \%$ & $94 \%$ & $100 \%$ \\
\hline Mean preop lesion vol $\left(\mathrm{cm}^{3}\right)$ & 34.8 & 21.4 & 8.1 & 1.3 \\
\hline Mean residual lesion vol $\left(\mathrm{cm}^{3}\right)$ & 24.3 & 8.7 & 0.4 & 0 \\
\hline No. of patients w/ ND at 3-mo FU & 0 & 1 & 1 & 0 \\
\hline $\begin{array}{l}\text { No. of patients w/ KPS deteriora- } \\
\text { tion at } 3 \text {-mo FU }\end{array}$ & 0 & 1 & 1 & 0 \\
\hline \multicolumn{5}{|l|}{ Glioma WHO grade III $(n=10) \ddagger$} \\
\hline No. of patients & 7 & 2 & 1 & \\
\hline EOR & $97 \%$ & $89 \%$ & $100 \%$ & \\
\hline Mean preop lesion vol $\left(\mathrm{cm}^{3}\right)$ & 36.4 & 25.8 & 30.7 & \\
\hline Mean residual lesion vol $\left(\mathrm{cm}^{3}\right)$ & 1.1 & 6.6 & 0 & \\
\hline No. of patients w/ ND at 3-mo FU & 2 & 0 & 0 & \\
\hline $\begin{array}{l}\text { No. of patients w/ KPS deteriora- } \\
\text { tion at } 3 \text {-mo FU }\end{array}$ & 2 & 0 & 0 & \\
\hline \multicolumn{5}{|l|}{ Glioma WHO grade IV $(n=41) \S$} \\
\hline No. of patients & 17 & 20 & 4 & \\
\hline EOR & $94 \%$ & $98 \%$ & $97 \%$ & \\
\hline Mean preop lesion vol $\left(\mathrm{cm}^{3}\right)$ & 35.9 & 33.6 & 14.3 & \\
\hline Mean residual lesion vol $\left(\mathrm{cm}^{3}\right)$ & 2.1 & 0.8 & 0.6 & \\
\hline No. of patients w/ ND at 3-mo FU & 2 & 1 & 0 & \\
\hline $\begin{array}{l}\text { No. of patients w/ KPS deteriora- } \\
\text { tion at 3-mo FU }\end{array}$ & 1 & 1 & 0 & \\
\hline \multicolumn{5}{|l|}{ Other $(n=11) \Uparrow$} \\
\hline No. of patients & 2 & 3 & 6 & \\
\hline EOR & $100 \%$ & $100 \%$ & $100 \%$ & \\
\hline Mean preop lesion vol $\left(\mathrm{cm}^{3}\right)$ & 2.1 & 19.7 & 10.2 & \\
\hline Mean residual lesion vol $\left(\mathrm{cm}^{3}\right)$ & 0 & 0 & 0 & \\
\hline No. of patients w/ ND at 3-mo FU & 0 & 0 & 1 & \\
\hline $\begin{array}{l}\text { No. of patients w/ KPS deteriora- } \\
\text { tion at } 3-\mathrm{mo} \text { FU }\end{array}$ & 0 & 0 & 0 & \\
\hline
\end{tabular}

$n=$ number of patients; ND = surgery-induced neurological deficit.

* Nine pilocytic astrocytomas, 1 angiocentric glioma, 1 subependymoma, 1 papillary glioneuronal lesion, 1 ganglioglioma.

† Four oligodendrogliomas, 6 mixed oligoastrocytomas, 6 diffuse astrocytomas, 1 central neurocytoma.

$\ddagger$ One anaplastic astrocytoma, 6 anaplastic oligoastrocytomas, 2 anaplastic oligodendrogliomas, 1 anaplastic ganglioglioma.

$\S$ Five diffuse midline gliomas, 36 glioblastomas.

II Six cavernous malformations, 1 arteriovenous malformation, 1 metastasis, 2 germinomas, 1 epithelial cyst.

$8(8.6 \%)$ had a ventriculoperitoneal shunt (VPS; all placed at other institutions), and 4 (4.3\%) had undergone endoscopic third ventriculocisternostomy (3VC; all performed at other institutions). 
Early postoperative problems in CSF circulation (within 3 months after surgery) occurred in 7 patients (7.6\%; 6/7 with high-grade glioma) who required VPS placement. Early postoperative CSF trouble developed in $14.6 \%$ of patients with high-grade gliomas but in only $3.3 \%$ of patients with low-grade lesions. Late postoperative trouble with CSF circulation occurred in another 5 patients $(5.4 \%$; 3 endoscopic 3VCs and 2 VPSs).

Intraoperatively, 12 patients, all undergoing an AIT approach, had an $\mathrm{m} 3 \mathrm{VC}$, while in 2 patients undergoing a PITS approach, the lamina terminalis could be opened. None of these patients required a VPS in the postoperative period, whereas 1 patient developed external hydrocephalus requiring a subduroperitoneal shunt. Six patients received an intraoperative external ventricular drain, which was then left in situ in the immediate postoperative course. In one of these cases, acute postoperative hydrocephalus developed because the external ventricular drain had failed and was eventually replaced.

\section{Discussion}

In this study, we present, to the best of our knowledge, the largest single-surgeon series of patients operated on for thalamic lesions in the contemporary era. All 92 patients underwent endoscope-assisted microsurgical removal according to a strict TCi or TCTV philosophy. In none of the cases was a transcortical approach adopted.

In the literature, the results of thalamic surgery are variable: the GTR rate ranges from $0 \%{ }^{16}$ to $81 \%{ }^{14}$ and permanent morbidity varies between $0 \%{ }^{16}$ and $84 \% .^{10}$ EOR is inconsistently reported. Several factors can be responsible for such variability: surgeon experience, different morbidity and GTR/EOR assessment criteria, patient and histopathological heterogeneity, and different surgical approaches. Our GTR and EOR rates compare favorably with those reported in the literature, and our complication rate is in line with those in modern series. It is worth mentioning, however, that our data originate from a prospective registry, a fact that notoriously leads to a higher number of registered complications than in retrospective studies.

We believe that the implementation of a TCi philosophy is crucial for surgical outcome. In a recent study, ${ }^{1}$ we illustrated the topographic anatomy of the thalamus, proposing segmentation of its free surface into lateral ventricle, velar, cisternal, and third ventricle surfaces. Each surface is delimited by clearly visible anatomical landmarks intraoperatively, which is essential for intraoperative orientation, and is readily identifiable if the surface is approached via a TCTV or TCi route. In this way, no unnecessary manipulation of healthy cerebral parenchyma is required.

Once the most appropriate thalamic surface is safely reached, removal of the targeted lesion is facilitated by the fact that thalamic lesions tend to remain localized within the thalamus. ${ }^{6,40}$ As reported by Yaşargil, ${ }^{6}$ thalamic lesions show expansive growth, which may even result in gross displacement of surrounding structures such as the IC or the hypothalamus without true infiltration. It follows that the landmarks delimiting the surgically accessible thalamic surfaces are usually preserved and can serve as reliable anatomical landmarks to guide safe resection. Moreover, as the surrounding anatomical structures are displaced and not infiltrated, they can be preserved. This is important in patients with lesions in close contact with the IC.

The IC is reportedly the most common source of morbidity. In our experience, risk of damage to the IC can be kept to a minimum if transcortical approaches are avoided, if a pure lesionectomy is done, and if the vascular supply to the IC is spared. Performing a pure lesionectomy requires correct discrimination between neoplastic and healthy tissue. This is not easy, particularly in cases of low-grade glioma, whose texture and consistency are often similar to those of the normal thalamus. However, high-grade neoplastic tissue usually has a looser structure than healthy tissue and thus can be more easily removed with suction at low power. Moreover, it is typically more prone to diffuse oozing. Surgical adjuncts such as ioUS and 3T-ioMR, as well as intraoperative neuromonitoring, are also helpful. Still, the borders of the tumor are difficult to recognize, and not infrequently a thin layer of tumor can remain, accounting for our 26\% NTRs and 8\% STRs. 3T-ioMR can be helpful for attaining GTR; however, differentiating residual tumor from edema or surgical contusion is not always straightforward. Damage to the IC can be avoided because of the different sources of vascularization of the thalamus and IC. The thalamus is fed by the posterior circulation (mainly branches of the posterior communicating artery), whereas the IC receives its supply mostly from the anterior circulation (anterior choroidal artery and lateral lenticulostriate arteries). ${ }^{40,41}$ This vascular border between the two territories implies that arterial hemostasis within the described anatomical limits of the thalamic surfaces can be safely performed without damaging the IC. However, the same does not apply to the venous system (particularly the thalamostriate vein), as its distribution pattern cannot be predicted. ${ }^{11,42}$

\section{Choice of Surgical Approach}

At our center, upfront biopsy (stereotactic) is indicated only if there is a high clinical-radiological suspicion of lymphoma or if the patient's medical condition contraindicates surgery. In all other cases, surgery is performed with the aim of the maximum possible EOR. Given the rarity of thalamic lesions and the peculiar challenges posed by every new patient, there can be no dogmatic algorithm for choosing the surgical approach. A flexible attitude and the readiness for smooth transitions from one approach to the other are required.

The AIT approach is our first choice if the tumor reaches the lateral ventricle surface and the patient presents with a dilated ventricle system. There are three reasons underlying this preference. First, if the ventricles are dilated, the intraventricular anatomy is easily recognizable, and thus surgical orientation and maneuverability are improved if coming from this route. The anatomical limits of the ventricular surface of the thalamus, particularly the thalamostriate vein and the stria terminalis, guide the resection since they mark the medial limit of the IC (thus the lateral limit of the resection). Second, through an AIT transforaminal approach, an $\mathrm{m} 3 \mathrm{VC}$ can often be done during the same procedure. Third, the AIT permits ioUS and 3TioMR. In patients in whom the lesion does not reach the 
lateral ventricle surface (as visible on preoperative coronal MRI sequences), the ventricles are small, or the required angle of attack is too steep, the AIT approach is not preferred, and a posterior pathway should be considered.

The PITS approach is an option for tumors displacing the splenium of the $\mathrm{CC}$ superiorly or extending sufficiently inferior to it, so that the splenium does not hinder the trajectory. If this is not the case, then another approach should be chosen, as blind traction of lesions protruding below the splenium, but mostly hidden beneath it, is highly dangerous and poses a risk to the internal cerebral veins. We do not recommend a posterior callosotomy for several reasons: first, it is not anatomically useful, as it gives access to the velum interpositum only; second, a high rate of neuropsychological deficits may follow it; and third, it endangers the internal cerebral veins and both fornices. The PITS approach offers some advantages: it allows m3VC through the lamina terminalis and 3T-ioMR. Lastly, it offers a fair view into the aqueduct in patients in whom the lesion pushes it downward, particularly if an angled optic is used.

The PeST approach is the only viable option to reach the cisternal surface of the thalamus if the lesion is hidden beneath the splenium. Given the need for atraumatic, gravity-mediated relaxation of the cerebellum, required for opening the transverse fissure, we always perform the PeST approach with the patient in the semisitting position, which in our setting does not imply relevant side effects. ${ }^{43}$ This approach also offers a relatively long reach, which is advantageous if the lesion has a significant anterosuperior extension, and at the same time it allows us to reach tumor extending into the mediobasal temporal region, as happened in 2 cases in our series. The disadvantage of the PeST approach is that it does not allow us to perform 3TioMR.

The PeCSS approach is best for those tumors confined within the third ventricle surface. It offers a pathway to the third ventricle without the need for fornix manipulation or damage to normal thalamic tissue. In our series, this approach was needed in only 3 patients and could be done without permanent morbidity. However, all patients who had undergone a PeCSS approach experienced a transient postoperative upward gaze palsy (which always resolved within 2 months), possibly due to manipulation of the posterior commissure. ${ }^{44}$ If the lesion reaches up to the tenia choroidea or spreads the choroidal fissure, then the AIT can be considered.

\section{CSF Disturbances}

Disturbances in CSF circulation are common in people with thalamic gliomas, either at presentation or sometime during the illness. Of our 92 patients, 29 (31.5\%) had hydrocephalus either pre- or postoperatively. A key role in the pathogenesis of hydrocephalus is played by the aqueduct, which can be either compressed by the lesion (directly or indirectly) or obstructed by surgical debris. To minimize that risk, we prefer not to leave any hemostatic agent in situ at the end of surgery. For the same reason, as well as for hemostasis, the ventricular system is continuously flushed for at least 30 minutes after lesion resection.

We also observed that perilesional tissues are frailer and softer in patients with high-grade glioma than in those with other lesions. Cerebral tissue compliance also seems to be altered, as suggested by some episodes in which the lateral ventricle collapsed postoperatively, resulting in an isolated temporal horn. It could be argued that the different consistency and compliance of tissues may lead to a postoperative collapse of the periaqueductal parenchyma, eventually leading to occlusion or subocclusion of CSF conduits and thereby possibly justifying the high rate of postoperative hydrocephalus in our patients with highgrade glioma (7/48 patients [14.6\%]).

Alternatively, the resection of a high-grade glioma may alter CSF protein content and lead to the development of communicating hydrocephalus, as suggested by the literature. ${ }^{45}$ Given our initial experience at the beginning of this series and based on the abovementioned considerations, we perform an $\mathrm{m} 3 \mathrm{VC}$ through the tuber cinereum or lamina terminalis on a routine basis whenever technically feasible.

\section{Operative Technical Adjuncts}

We applied several technological tools pre- and intraoperatively. ioUS is useful for intraoperative guidance and for choosing the point for callosotomy. ${ }^{37}$ However, it cannot be used for patients in the semisitting position and is less helpful than 3T-ioMR for resection control. 3T-ioMR permits excellent intraoperative resection control and allows the surgeon to take fewer risks during resection. However, it cannot be used if the semisitting position is required. The endoscope helps at the very beginning of the procedure, just before attacking the lesion, to study surgical anatomy, and at the end of resection to inspect the surgical field, check for EOR and hemostasis around blind corners, and ensure patency of the aqueduct. Intraoperative navigation as well as intraoperative fluorescence did not prove to be helpful. Intraoperative neuromonitoring was routinely used, but in our hands, it never proved to be crucial for resection.

In our experience, these technological improvements represent a means to increase the surgeon's confidence and possibly refine the surgical outcome, provided that the mainstays of neurosurgery are always respected. Exact anatomical knowledge ${ }^{1}$ helps in accurate topographical diagnosis. This, in turn, is fundamental for determining appropriate surgical indications and, as we showed, for a carefully weighed choice of the surgical approach. These three theoretical pillars are then prerequisites for precise microsurgical skills to successfully finalize surgery.

\section{Conclusions}

Endoscope-assisted microsurgery permits the safe removal of thalamic lesions with an acceptable risk of morbidity. In our experience, virtually all thalamic lesions can be accessed through one of the four free thalamic surfaces (lateral ventricle, velar, cisternal, and third ventricle surface) via either a TCi or a TCTV approach. These approaches facilitate optimal intraoperative orientation and access to one or more of the four thalamic surfaces without unnecessary manipulation of normal brain parenchyma. Patients harboring high-grade gliomas seem to be 
more likely to develop a postoperative disturbance of CSF circulation. For this reason, the AIT approach should be favored whenever feasible, as it permits the surgeon to easily perform an $\mathrm{m} 3 \mathrm{VC}$.

\section{Acknowledgments}

This study was kindly supported by Miss Nur Atabay. We also thank Julie Yamamoto, MA, for editorial assistance. We would also like to acknowledge Mr. Peter Roth for his outstanding artistic work.

\section{References}

1. Serra C, Türe U, Krayenbühl N, et al. Topographic classification of the thalamus surfaces related to microneurosurgery: a white matter fiber microdissection study. World Neurosurg. 2017:97:438-452.

2. Serra C, Guida L, Staartjes VE, et al. Historical controversies about the thalamus: from etymology to function. Neurosurg Focus. 2019;47(3):E13.

3. Schmahmann JD. Vascular syndromes of the thalamus. Stroke. 2003;34(9):2264-2278.

4. Lo WB, Rutka JT. Thalamic gliomas. In: Tonn J-C, Reardon DA, Rutka JT, Westphal M, eds. Oncology of CNS Tumors. Springer; 2019:459-480.

5. Esquenazi Y, Moussazadeh N, Link TW, et al. Thalamic glioblastoma: clinical presentation, management strategies, and outcomes. Neurosurgery. 2018;83(1):76-85.

6. Yaşargil MG. Microneurosurgery. Vol IVB. Thieme; 1996.

7. Rangel-Castilla L, Spetzler RF. The 6 thalamic regions: surgical approaches to thalamic cavernous malformations, operative results, and clinical outcomes. J Neurosurg. 2015; 123(3):676-685.

8. Chang EF, Gabriel RA, Potts MB, et al. Supratentorial cavernous malformations in eloquent and deep locations: surgical approaches and outcomes. Clinical article. J Neurosurg. 2011;114(3):814-827.

9. Bertalanffy H, Benes L, Miyazawa T, et al. Cerebral cavernomas in the adult. Review of the literature and analysis of 72 surgically treated patients. Neurosurg Rev. 2002;25(1-2): $1-55$.

10. Arseni C. Tumors of the basal ganglia; their surgical treatment. AMA Arch Neurol Psychiatry. 1958;80(1):18-24.

11. McKissock W, Paine KW. Primary tumours of the thalamus. Brain. 1958;81(1):41-63.

12. Tovi D, Schisano G, Liljeqvist B. Primary tumors of the region of the thalamus. J Neurosurg. 1961;18:730-740.

13. Cheek WR, Taveras JM. Thalamic tumors. J Neurosurg. 1966;24(2):505-513.

14. Hirose G, Lombroso CT, Eisenberg H. Thalamic tumors in childhood. Clinical, laboratory, and therapeutic considerations. Arch Neurol. 1975;32(11):740-744.

15. Bernstein M, Hoffman HJ, Halliday WC, et al. Thalamic tumors in children. Long-term follow-up and treatment guidelines. J Neurosurg. 1984;61(4):649-656.

16. Hoffman HJ, Soloniuk DS, Humphreys RP, et al. Management and outcome of low-grade astrocytomas of the midline in children: a retrospective review. Neurosurgery. 1993;33(6) 964-971.

17. Cuccia V, Monges J. Thalamic tumors in children. Childs Nerv Syst. 1997;13(10):514-521.

18. Steiger HJ, Götz C, Schmid-Elsaesser R, Stummer W. Thalamic astrocytomas: surgical anatomy and results of a pilot series using maximum microsurgical removal. Acta Neurochir (Wien). 2000;142(12):1327-1337.

19. Özek MM, Türe U. Surgical approach to thalamic tumors Childs Nerv Syst. 2002;18(8):450-456.

20. Albright AL. Feasibility and advisability of resections of tha- lamic tumors in pediatric patients. J Neurosurg. 2004;100(5) (Suppl Pediatrics):468-472.

21. Baroncini M, Vinchon M, Minéo JF, et al. Surgical resection of thalamic tumors in children: approaches and clinical results. Childs Nerv Syst. 2007;23(7):753-760.

22. Moshel YA, Link MJ, Kelly PJ. Stereotactic volumetric resection of thalamic pilocytic astrocytomas. Neurosurgery. 2007;61(1):66-75.

23. Puget S, Crimmins DW, Garnett MR, et al. Thalamic tumors in children: a reappraisal. J Neurosurg. 2007;106(5)(suppl): 354-362.

24. Kramm CM, Butenhoff S, Rausche U, et al. Thalamic highgrade gliomas in children: a distinct clinical subset? Neuro Oncol. 2011;13(6):680-689.

25. Sai Kiran NA, Thakar S, Dadlani R, et al. Surgical management of thalamic gliomas: case selection, technical considerations, and review of literature. Neurosurg Rev. 2013;36(3): 383-393.

26. Bilginer B, Narin F, Işıkay I, et al. Thalamic tumors in children. Childs Nerv Syst. 2014;30(9):1493-1498.

27. Cao L, Li C, Zhang Y, Gui S. Surgical resection of unilateral thalamic tumors in adults: approaches and outcomes. BMC Neurol. 2015;15:229.

28. Wong TT, Chen HH, Liang ML, et al. Clinical considerations and surgical approaches for low-grade gliomas in deep hemispheric locations: thalamic lesions. Childs Nerv Syst. 2016; 32(10):1895-1906.

29. Zheng $\mathrm{X}, \mathrm{Xu} \mathrm{X}$, Zhang $\mathrm{H}$, et al. A preliminary experience with use of intraoperative magnetic resonance imaging in thalamic glioma surgery: a case series of 38 patients. World Neurosurg. 2016;89:434-441.

30. Wu B, Tang C, Wang Y, et al. High-grade thalamic gliomas: microsurgical treatment and prognosis analysis. J Clin Neurosci. 2018;49:56-61.

31. Kim JH, Phi JH, Lee JY, et al. Surgical outcomes of thalamic tumors in children: the importance of diffusion tensor imaging, neuro-navigation and intraoperative neurophysiological monitoring. Brain Tumor Res Treat. 2018;6(2):60-67.

32. Cinalli G, Aguirre DT, Mirone G, et al. Surgical treatment of thalamic tumors in children. J Neurosurg Pediatr. 2018;21(3): 247-257.

33. Steinbok P, Gopalakrishnan CV, Hengel AR, et al. Pediatric thalamic tumors in the MRI era: a Canadian perspective. Childs Nerv Syst. 2016;32(2):269-280.

34. Akiyama O, Matsushima K, Gungor A, et al. Microsurgical and endoscopic approaches to the pulvinar. J Neurosurg. 2017;127(3):630-645.

35. Harput MV, Gonzalez-Lopez P, Türe U. Three-dimensional reconstruction of the topographical cerebral surface anatomy for presurgical planning with free OsiriX software. Neurosurgery. 2014;10(suppl 3):426-435.

36. Türe U, Yasargil MG, Al-Mefty O. The transcallosal-transforaminal approach to the third ventricle with regard to the venous variations in this region. J Neurosurg. 1997;87(5): $706-715$

37. Goga $C$, Türe $U$. The anterior transcallosal approach to a cerebral aqueduct tumor: a 3-dimensional operative video. Neurosurgery. 2014;10(suppl 3):492.

38. Harput MV, Türe U. Microneurosurgical removal of a posterior thalamic glioma via posterior interhemispheric subsplenial approach in lateral oblique position. Oper Neurosurg (Hagerstown). 2017;13(5):643.

39. Keles A, Harput MV, Türe U. Endoscope-assisted contralateral perimedian supracerebellar suprapineal approach to the third ventricle surface of the thalamus: 3-dimensional operative video. Oper Neurosurg (Hagerstown). 2020;19(3):E306E307.

40. Yaşargil MG. Microneurosurgery. Vol IVA. Thieme; 1994.

41. Schlesinger B. The Upper Brainstem in the Human: Its Nu- 
clear Configuration and Vascular Supply. Springer-Verlag; 1976.

42. Salamon G, Huang YP. Radiologic Anatomy of the Brain. Springer-Verlag; 1976.

43. Türe H, Harput MV, Bekiroğlu N, et al. Effect of the degree of head elevation on the incidence and severity of venous air embolism in cranial neurosurgical procedures with patients in the semisitting position. J Neurosurg. 2018;128(5):15601569.

44. Bender MB. Brain control of conjugate horizontal and vertical eye movements: a survey of the structural and functional correlates. Brain. 1980;103(1):23-69.

45. Montano N, D’Alessandris QG, Bianchi F, et al. Communicating hydrocephalus following surgery and adjuvant radiochemotherapy for glioblastoma. J Neurosurg. 2011;115(6): 1126-1130.

\section{Disclosures}

The authors report no conflict of interest concerning the materials or methods used in this study or the findings specified in this paper.

\section{Author Contributions}

Conception and design: U Türe, Serra. Acquisition of data: U Türe, Serra, Yaltırık, Harput. Analysis and interpretation of data: U Türe, Serra, H Türe, Yaltırık. Drafting the article:
U Türe, Serra, H Türe, Yaltırık. Critically revising the article: U Türe, Serra, Yaltırık, Harput. Reviewed submitted version of manuscript: all authors. Approved the final version of the manuscript on behalf of all authors: U Türe. Statistical analysis: Serra, Yaltırık. Administrative/technical/material support: U Türe, Serra, Harput. Study supervision: U Türe. Performed all surgeries: U Türe.

\section{Supplemental Information}

Videos

Video 1. https://vimeo.com/427859155.

Video 2. https://vimeo.com/427860242.

\section{Correspondence}

Uğur Türe: Yeditepe University School of Medicine, Yeditepe University Kosuyolu Hospital, Istanbul, Turkey. drture@yahoo. com. 